\title{
Straight Path Handling Anomalies of Passenger Cars Induced by Suspension Component and Assembly Tolerances
}

\author{
M. De Rosa ${ }^{1}$, A. De Felice ${ }^{2}$, P. Grosso ${ }^{2}$ and S. Sorrentino ${ }^{2 *}$ \\ ${ }^{1}$ Product Development, Maserati SpA, \\ Via Emilia Ovest 911, 41123, Modena, Italy \\ ${ }^{2}$ Department of Engineering Enzo Ferrari, University of Modena and Reggio Emilia, \\ Via Vivarelli 10, 41125, Modena, Italy \\ *Email: silvio.sorrentino@unimore.it \\ Phone: +390592056307
}

\begin{abstract}
The effects on handling due to suspension component and assembly tolerances are analysed focusing on three anomalies affecting passenger cars travelling on straight paths, namely 'pull to side', 'steering wheel misalignment' and 'drift leeward'. These anomalies do not constitute safety problems but represent a cost factor in the automotive industry since they occur in small but not negligible percentage of the overall car production. The present contribution is therefore focused on understanding which are their causes, and on reducing their extent, in the specific case of vehicles with front Double wishbone suspension and rear Five arms suspension. To this purpose, the vehicle assembly process is analysed in terms of errors and tolerances for identify the most influential parameters on handling irregularities. Sensitivity analysis by multibody virtual modelling, and subsequent correlation with experimental data from pre-setting and wheel aligner benches, have provide sufficient information for setting tolerance thresholds, able to keep the handling anomalies under study within acceptable bounds. As a result, 'pull to side' and 'steering wheel misalignment' has been found to be mainly related to the set up phase of wheel angles (front camber angles influencing pull to side, front and rear toe angles influencing steering wheel misalignment), while the main cause of 'drift leeward' has been identified with (rear) ride steer. Application of the procedure to a production line led to a consistent reduction (from $3 \%$ down to about $1 \%$ of the overall production) of anomalous vehicles.
\end{abstract}

Keywords: Handling; suspension geometry; pull to side; steering wheel misalignment; drift leeward

\section{INTRODUCTION}

Among handling anomalies affecting passenger cars travelling on a straight path, the "pull to side' (PTS, lateral deviations from straight trajectory with steering wheel correctly aligned), the 'steering wheel misalignment' (SWM, straight trajectory with steering wheel not aligned) and the 'drift leeward' (DL, lateral deviations due to the effects of uneven ground surface on the rear suspensions) are particularly relevant. Even though they do not constitute safety problems, however, they represent a cost factor in the automotive industry, occurring in a small but not negligible percentage of the overall car production.

Several studies are devoted to investigating the contribution of tyre characteristics on the pull to side and steering-wheel misalignment (also referred to simply as drift). A 
model for describing steering pull as an effect of asymmetrical tyre cornering properties is discussed in [1], analysing the effects of tyre lateral force and aligning torque symmetries on a car's straight-line stability for both fixed and free control, and validated with free control data obtained on the road. In [2] a laboratory method is described for measuring residual aligning torque, which is presented as a predictor of tyre contribution to steering pull; test data are compared with respect to conicity levels on tyre combinations with different aligning torques. Tyre-induced pull and drift problems are respectively discussed also in [3-5], with special emphasis on plysteer, and [6-7]. A specific analytical model is developed in [8], aimed at investigating the effects of tyre characteristics, in particular plysteer and conicity, on making the longitudinal axis of road vehicles non-parallel to road axis during straight-ahead running.

On the other hand, the literature aimed at investigating the contribution of suspension geometry (more precisely, of tolerances of suspension systems) on the pull to side- and steering-wheel misalignment, is less extensive. The analysis presented in [9] is focused on a simulation-based dimensional tolerance optimization process, to minimize vehicle pulls by reduction of dimensional variation in a front suspension system. A computational method is proposed in [10] to evaluate the impact of dimensional variations on vehicle steering pull, in the specific case of front Mac Pherson suspension and rear Twist bar suspension. In [11] an analytical vehicle model and a full-vehicle model are considered, for studying the effect of suspension geometry on steering pull and drift (i.e. steering wheel misalignment). A robust design optimization method to reduce steering pull phenomenon is presented in [12], considering the tolerance of suspension system elements, such as hardpoints, spring, damper and bushings.

Clearly, these issues also have an impact on the design of control systems, such as active front steering control [13], as well as on steering performance of classes of vehicles, like autonomous drive vehicles [14] and ultra-light cars [15]. The present contribution is focused on understanding which is the causes and on reducing the extents of PTS, SWM and DL as handling anomalies due to suspension component and assembly tolerances, in the specific case of vehicles with front Double wishbone suspension and rear Five arms suspension. To this purpose, the vehicle assembly process is first analysed in terms of errors and tolerances by means of a design of experiment analysis (DOE), aimed at identifying which parameters can generate PTS, SWM and DL. Sensitivity analysis by multibody virtual modelling is then performed, for understanding how and at which level the relevant suspension parameters can affect the straight path handling of the vehicles under study. Finally, the results of simulations are correlated with experimental data from pre-setting and wheel aligner benches, for setting tolerance thresholds able to keep the anomalies under study within acceptable bounds. The expected result of this analysis is a relevant reduction of the percentage of vehicles affected by these handling anomalies on a whole production line.

\section{METHOD 1: DOE ANALYSIS OF THE ASSEMBLY PROCESS}

Two vehicle models have been considered, with suspension systems of the same typology. A design of experiment (DOE) analysis has been performed aimed at understanding the role of tolerances on specific parameters that can affect handling behaviour. More in detail, displacements of the suspension hardpoints with respect to their ideal position (in a chassis reference system) have been estimated as functions of combined tolerances due to both component tolerances and coupling tolerances. This analysis does not provide any information about how suspension parameters affect 
handling behaviour, nevertheless it yields realistic ranges of variation, which are necessary for handling analysis.

\section{Influential Parameters}

A two-wheel steering vehicle travels on a straight path if the average of slip angles at the front axle is equal to that at the rear axle, or, in other words, if the slip angle of the front axle and the slip angle of the rear axle in a single-track model of the vehicle are equal [16]. Generally, slip angles differ from toe angles because of tyre deformation. For this reason, in this study the crab angle $\left(\delta_{t}\right)$ of an axle is considered, representing the contribution to the axle slip angle given by the toe angles of the two wheels (say, $\delta_{1}$ and $\delta_{2}$ ):

$$
\delta_{t}=\frac{\delta_{1}-\delta_{2}}{2}
$$

where $\delta_{t}$ takes the sign of $\delta_{l}$. While on the rear axle the toe angles are imposed with respect to the mechanical symmetry axis (i.e. the axis passing by the front axle centre and the rear axle centre, which in general is not contained in the vehicle symmetry plane), the front toe angles are imposed with respect to the (rear) crab axis (defined by the rear toe angles), in order to reduce the differences between the front and rear axle slip angles. Clearly, PTS, SWM and DL are strongly related to the axle slip angles. PTS can occur only if the front and rear axle slip angles are not equal. SWM depends by different front and rear axle slip angles as well; in this case, however, the vehicle reacts to lateral forces due to the tyres by modifying the position of the front wheels until the front axle slip angle coincides with the rear one; consequently, the steering wheel changes its position, producing misalignment. DL can be evaluated as a dynamic variation of the rear axle slip angle, due to a vertical parallel excitation of the axle (Figure 1).

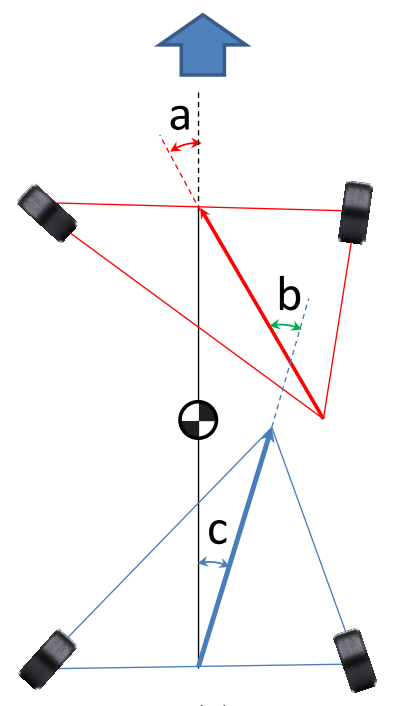

(a)

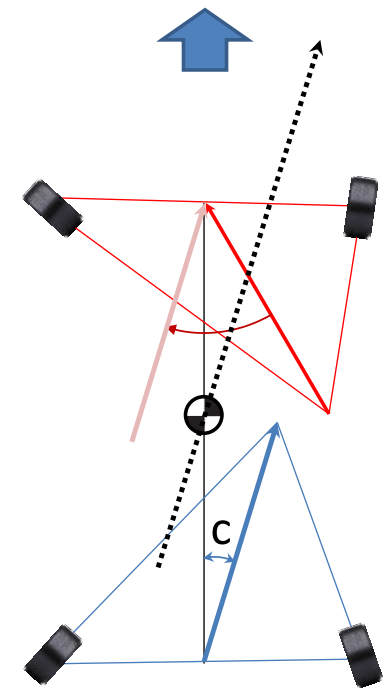

(b)

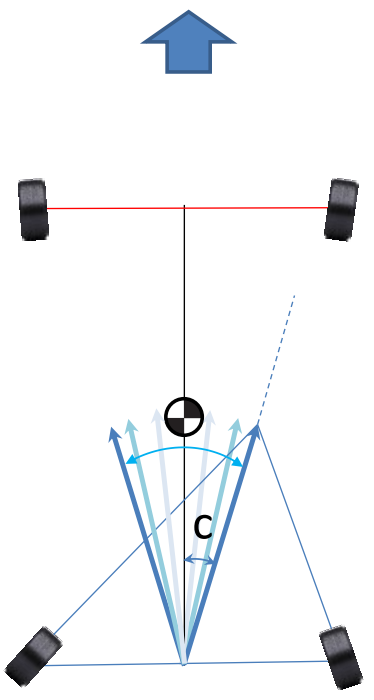

(c)

Figure 1. (a) Crab axis ( $\mathrm{a}=$ front crab angle; $\mathrm{b}=$ total crab angle; $\mathrm{c}=$ rear crab angle), (b) SWM effect and (c) DL effect.

The front and rear axle slip angles are also influenced by axle positioning on the vehicle's body during the mounting phase, and by different loads acting on the wheels of 
each axle. Therefore, vehicle's handling during straight path manoeuvres is affected by non-symmetric values (on the same axle) of the following parameters of interest for this study (geometric and dynamic): toe angles, camber angles, lateral displacement of wheel centre, vertical loads acting on wheel, ride steer behaviour in vertical travel.

\section{Suspension Assembly Process}

At the front axle the vehicles considered in this analysis mount Double wishbone suspensions [17], as shown in Figure 2 (a). By considering rigid elements and joints, the number of parameters characterizing cinematically this suspension mechanism is 19 . The mechanism is composed by upper control arm (upper wishbone), connected directly to vehicle body via two bushings; front subframe, connected to vehicle body via screws, lower control arm (lower wishbone), connected to front subframe via two bushings (connections with lateral regulation, for camber regulation and for solving tolerance problems related to single components); steering box, connected to front subframe via two screws; upright, connected to upper and lower control arms via two spherical joints; tie rod, connected to upright and steering box via two spherical joints.

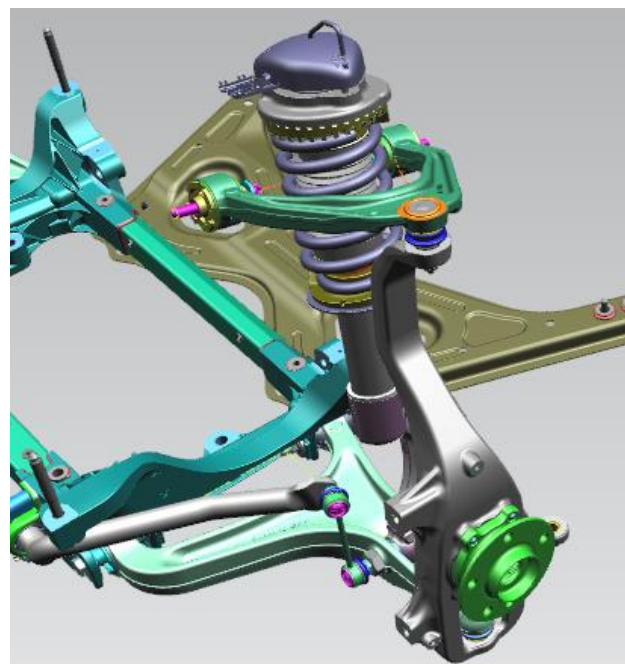

(a)

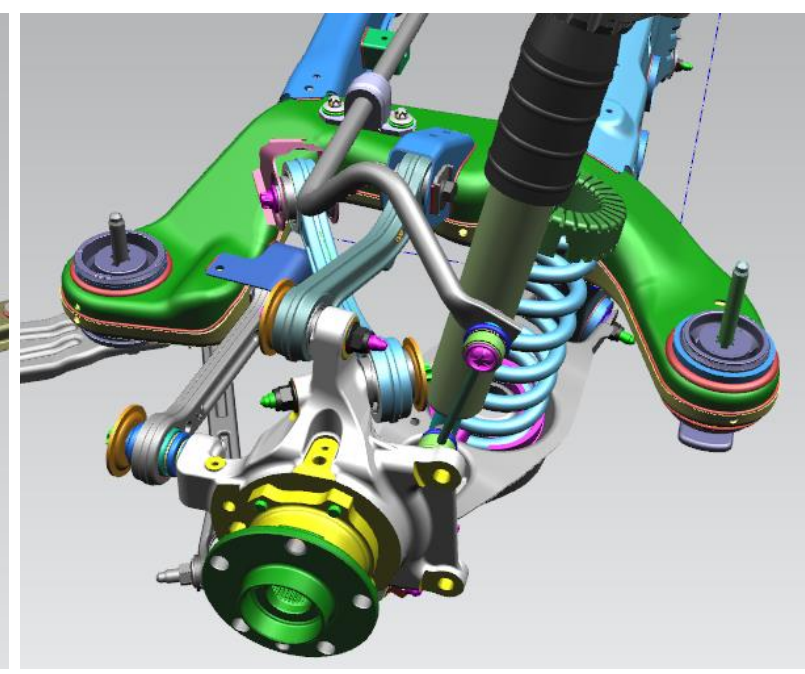

(b)

Figure 2. Front Double wishbone (a) and rear Five arms (b) suspensions.

The front suspension assembly process starts with the positioning of the front subframe on a presetting bench. The mounting sequence consists of the following steps: connection of lower control arm and steering box on subframe; connection of wheel assembly (upright, hub, and brake system) on the lower control arm; connection of upper control arm on the chassis; mounting of subframe on the chassis and connection of the upright to the upper control arm (coupling phase).

During this process, the assembly anomalies that can be generated are: nonsymmetric king pin axes; non-symmetric caster, camber and toe angles (the last two anomalies are generally adjusted in the final phase of the assembly process); nonsymmetric lateral and/or angular (around a vertical axis) positioning of subframe with respect to chassis (compromising symmetry of the suspension mechanisms at the two sides of the same axle); different ratios between spring travel and wheel travel. 
A DOE analysis has been performed on the front suspension. The influence on suspension parameters of hardpoint displacements due to worst combination of tolerances are represented by histograms as those displayed in Figure 3 (including numbered hardpoints, with displacements expressed in a chassis XYZ reference system; in the histograms, the percentages are defined with respect to the total variation of the parameter under study: ride steer or ride camber). Among the most influential parameters, ride steer in Figure 3(a) is affected mainly by the Z (vertical) displacements of hardpoints 3 (lower control arm) and 2 (steering box), while the largest variation on ride camber is given by the $\mathrm{Z}$ displacements of hardpoints 7 and 8 (upper control arm).
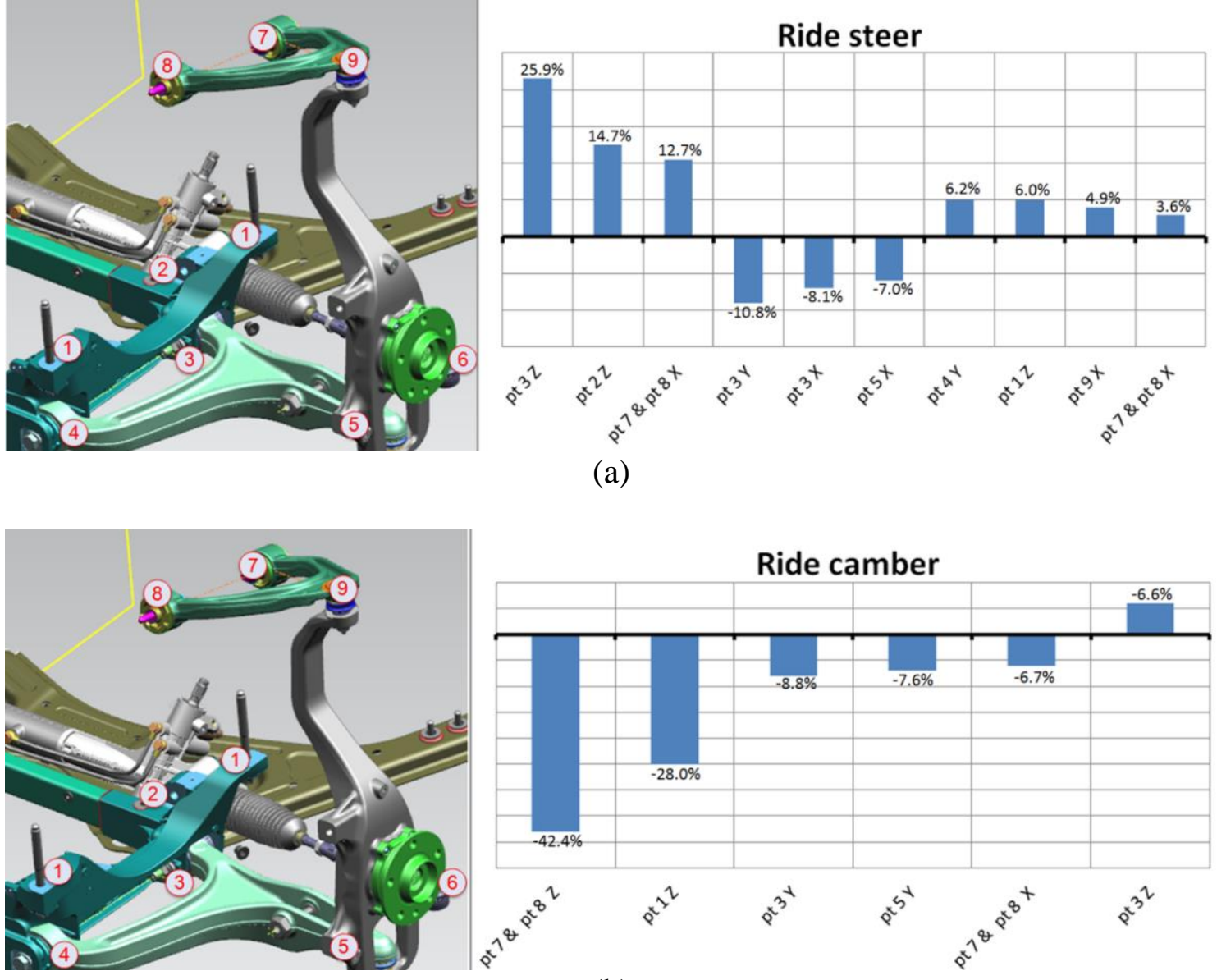

(b)

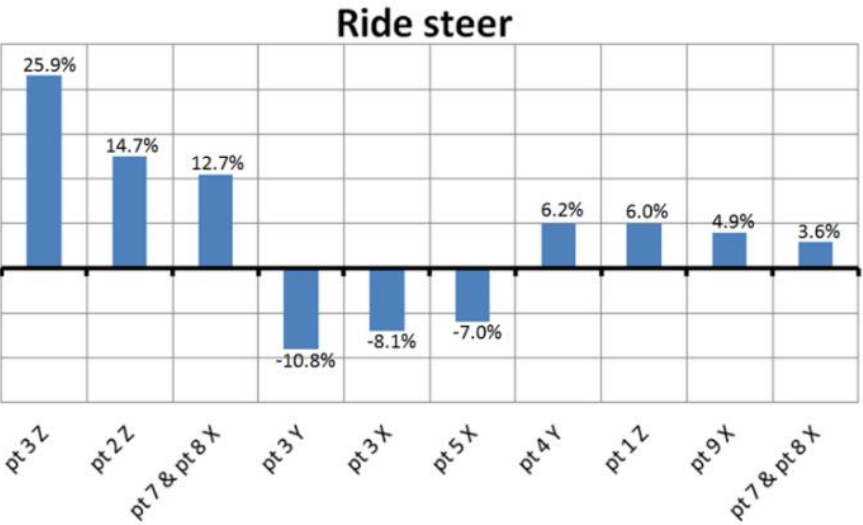

(a)

Figure 3. Front suspension: (a) ride steer and (b) ride camber.

At the rear axle the vehicles considered in this analysis mount multilink suspensions (Five arms [17]), as shown in Figure 2(b). By considering rigid elements and joints, the number of parameters characterizing cinematically this suspension mechanism is 29 . In particular, the mechanism comprises two specific links respectively dedicated to camber and toe regulation.

The rear suspension assembly process is a delicate matter: regulation buttonholes are necessary to correctly complete the coupling phase procedure. Also in this case the subframe is first positioned on a pre-setting bench. The mounting sequence consists of the following steps: mounting of all the links on the subframe with screw positioning; positioning of wheel assembly on the bench and connection to the arms via screws; tightening of the screws in their buttonholes. 
A more complex DOE than that performed for the front suspension leads to histograms like the one represented in Figure 4, showing the contribution of rear suspension tolerances on the ride steer (percentages defined with respect to the total variation of ride steer). The latter parameter is affected mainly by $\mathrm{Z}$ (vertical) displacement between hardpoints 6 and 11 (tie rod hardpoints).
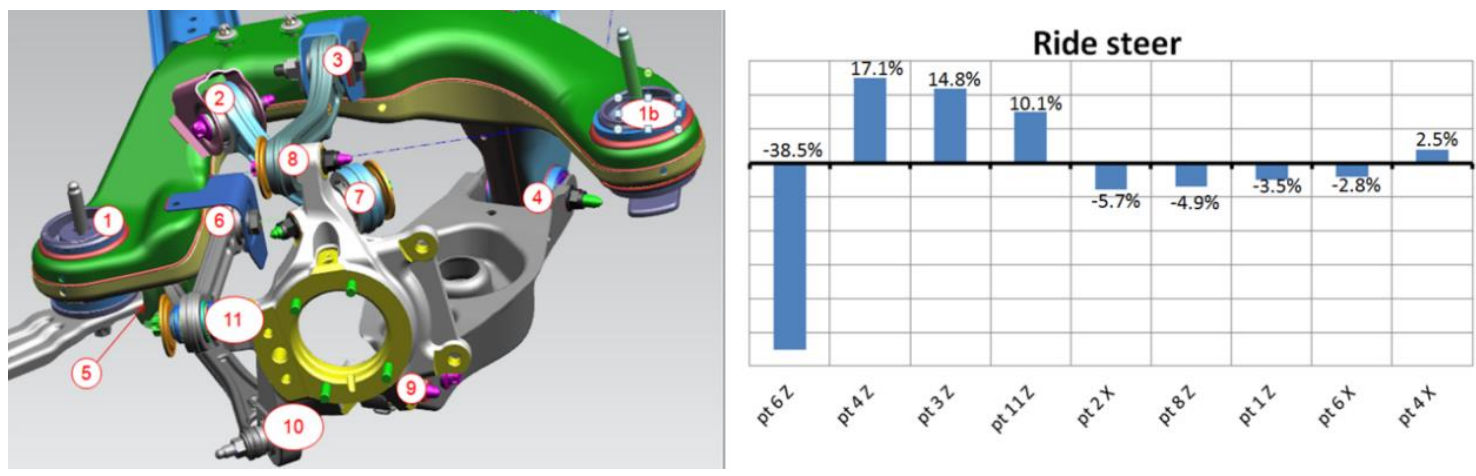

Figure 4. Rear suspension: ride steer.

\section{Full Vehicle Assembly Process}

Chassis, front and rear subframes are the main subsystems composing a full vehicle. They are connected during the coupling phase. Chassis can be affected by mass distribution anomalies (generating non-symmetric downforces on the wheels on opposite sides of the vehicle) and by geometric anomalies (twisting and bending; only the former are relevant within the present analysis, since they can cause different preloads on the opposite wheels of the same axle, and consequently different travel positions of the two suspensions).

During the subframe assembly process, there are two critical phases, potentially generating the anomalies under study: coupling phase and wheel alignment (last phase of the assembly process, in which a vehicle complete in all its parts is positioned on the wheel aligner bench for setting the toe and camber angles of the front and rear axles).

If the chassis is symmetry and not twisted, possible errors during coupling phase can lead to: axle centre out of the chassis symmetry plane; non-parallel axles in lateral direction. On the other hand, during wheel alignment, possible errors can originate from incorrect wheel scanning on the aligner bench (the laser scanning process identify the wheel centres, along with toe and camber angles; from wheel centres, the positions of the two axles are determined, and consequently the vehicle mechanical symmetry plane, which, depending on the coupling phase phase, not necessarily coincides with the chassis symmetry plane). During the final setting of toe and camber angles, attention must be paid in avoiding any possible contribution yielding preloads on the elastic components, which after regulation would affect the setup.

\section{METHOD 2: VIRTUAL SENSITIVITY ANALYSIS}

On the basis of the results obtained from DOE analysis, a virtual sensitivity multibody analysis has been performed, aimed at understanding how and at which level the relevant suspension parameters can affect the handling on a straight path of the vehicles under study.

\section{Model Definitions and Input Simulations}


A vehicle multibody model has been created using a commercial software (MSC Adams Car). Straight line manoeuvres have been simulated at an imposed vehicle speed of 100 $\mathrm{km} / \mathrm{h}$. Both a perfectly flat ground surface and a standard highway surface profile have been considered, the latter for taking into account vertical excitation due to irregularities of the ground. Notice that, adopting a highway surface profile, small lateral displacements can occur even in the case of a completely symmetric vehicle, due to different vertical inputs at each side of the car. On the other hand, a perfectly flat ground surface has been adopted to evaluate the anomalies due to vehicle process only; for PTS contribution, the steering wheel has been locked to $0^{\circ}$; while for SWM contribution, the steering wheel has been set free to rotate.

To evaluate the effects of asymmetry involving toe angles (front toe with respect to crab axis), camber angles, and lateral displacement of wheel centres, different hardpoint configurations have been considered, in each case imposing the maximum tolerance differences found by DOE analysis; for vertical load asymmetry, different preloads have been imposed on the springs of the same axle; for different ride steer on the two wheels of the rear axle, the worst case evaluated with DOE analysis has been considered (largest error due to component tolerances), yielding different hard-point positions, and consequently different elasto-kinematic behaviours of the two rear suspensions.

\section{Virtual Simulation Results}

Vertical load asymmetry on the front axle suspensions has been simulated by imposing a $6 \%$ difference in nominal preload on the two front springs, causing a displacement of centre of gravity from the symmetry plane, and consequently a lateral deviation of the vehicle (less than $5 \mathrm{~mm}$ over a $300 \mathrm{~m}$ travel) and a rotation of the steering wheel (lower than $2 \%$ ), as shown in Figure 5(a) and 5(b) respectively.

Asymmetry due to front subframe coupling phase errors has been simulated by imposing lateral displacements of lower control arms, and consequently of wheel centres (notice that after creating this asymmetry, camber and toe angles have been perfectly regulated). The effects due to maximum tolerances on PTS as in Figure 6(a) and SWM shown in Figure 6(b) are very small.

Asymmetry in wheel angles setup phase (camber and toe) is the most important one for investigating the anomalies under study. As shown in Figure 7(a), an asymmetrical regulation of the front camber angle produces the highest PTS effect on the vehicle (in fact, at low slip angles, camber variations are particularly effective on tyre lateral forces). In regards to SWM, the most influential parameters have been found to be the front and rear toe angles, which strongly affect the sideslip, as shown in Figure 7(b).

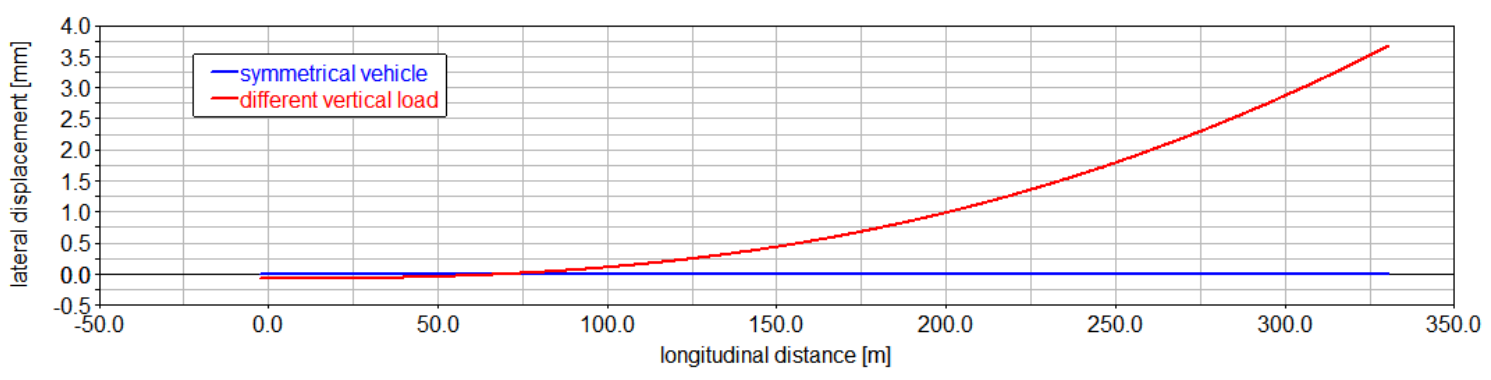

(a) 


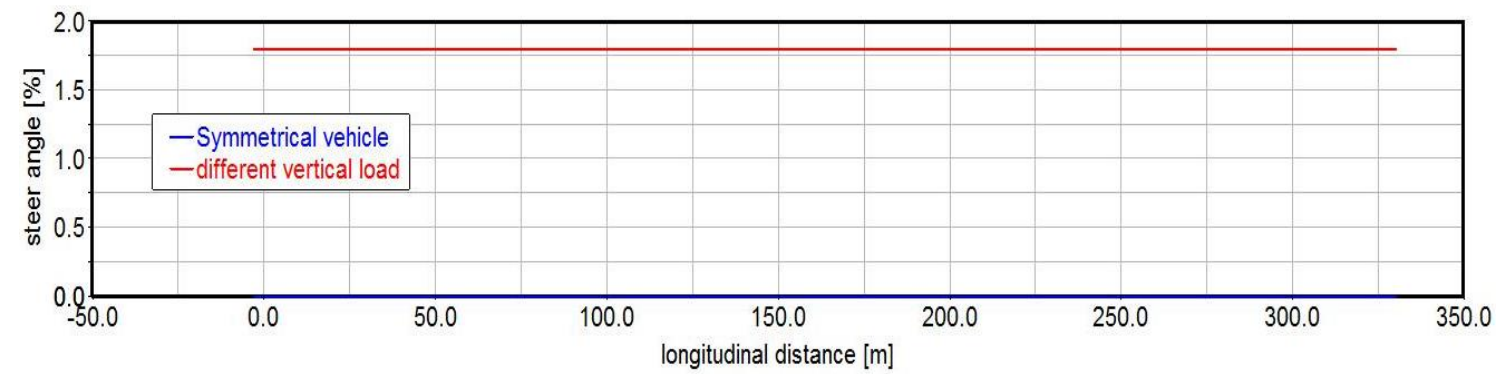

(b)

Figure 5. Effect of a vertical load asymmetry in front axle suspensions on lateral deviation (a) and steering wheel rotation (b).

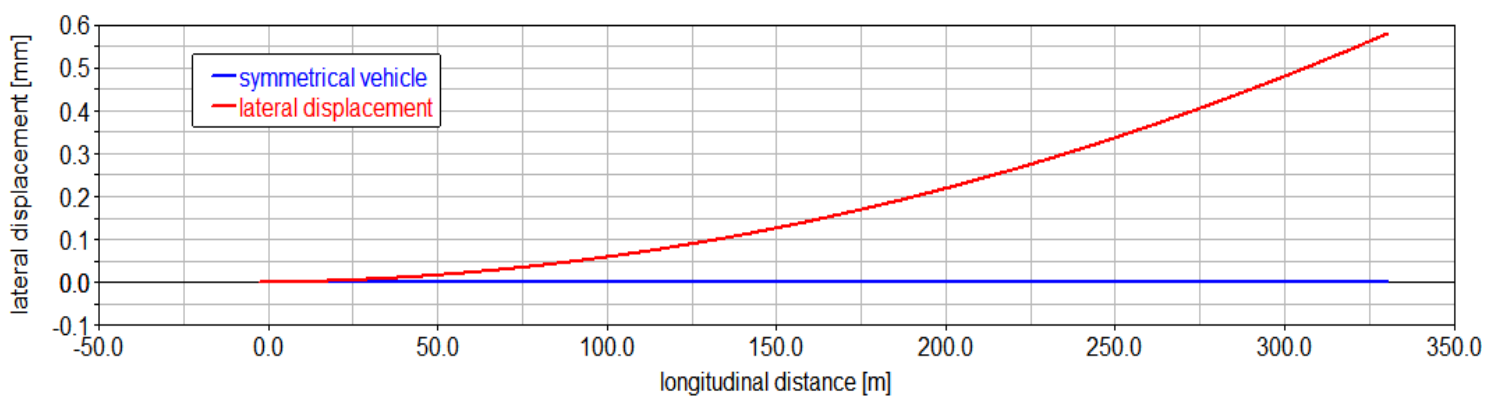

(a)

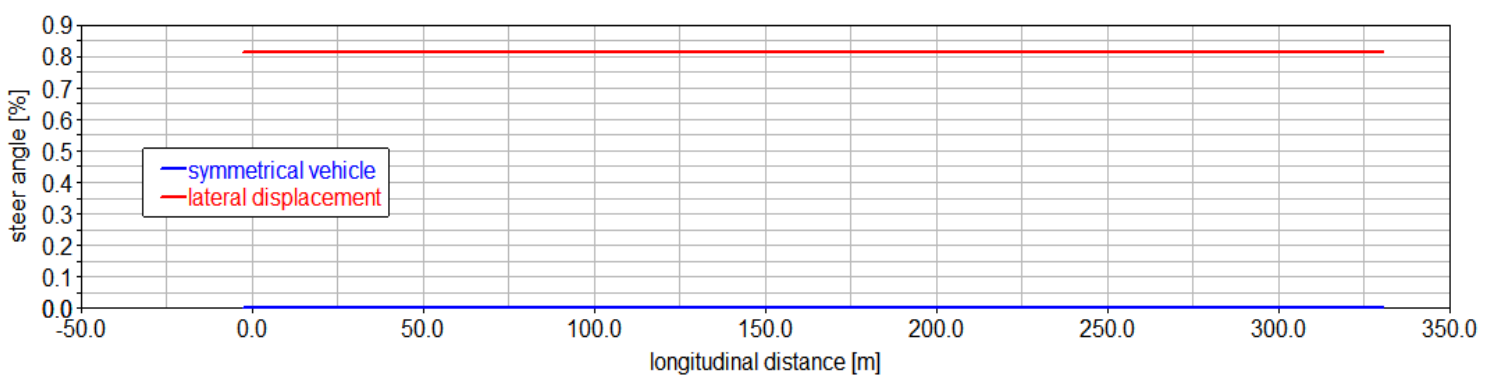

(b)

Figure 6. Effect of asymmetric lateral displacements of front lower control arms on lateral deviation (a) and steering wheel rotation (b).

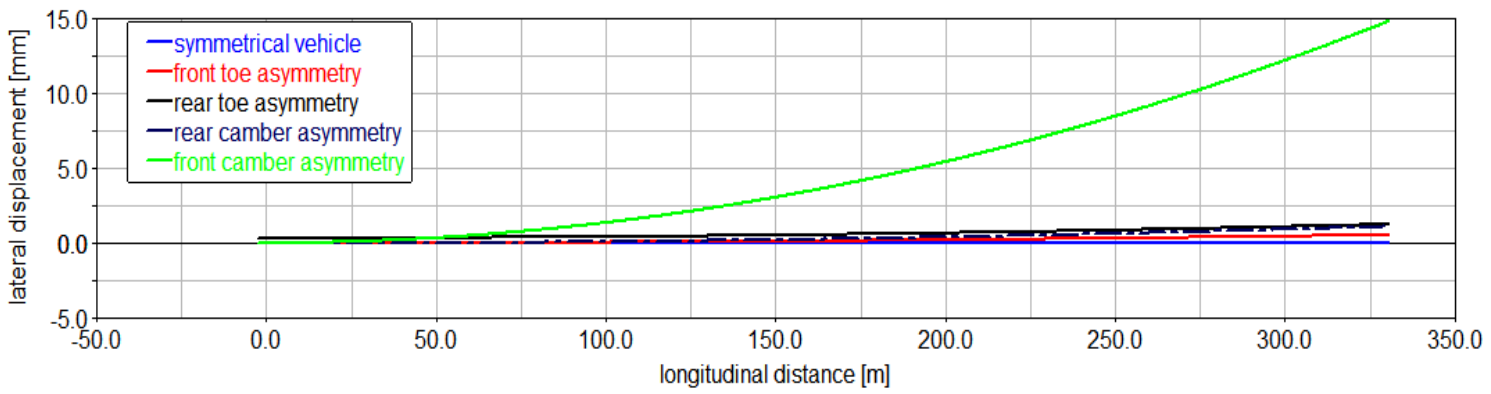

(a) 


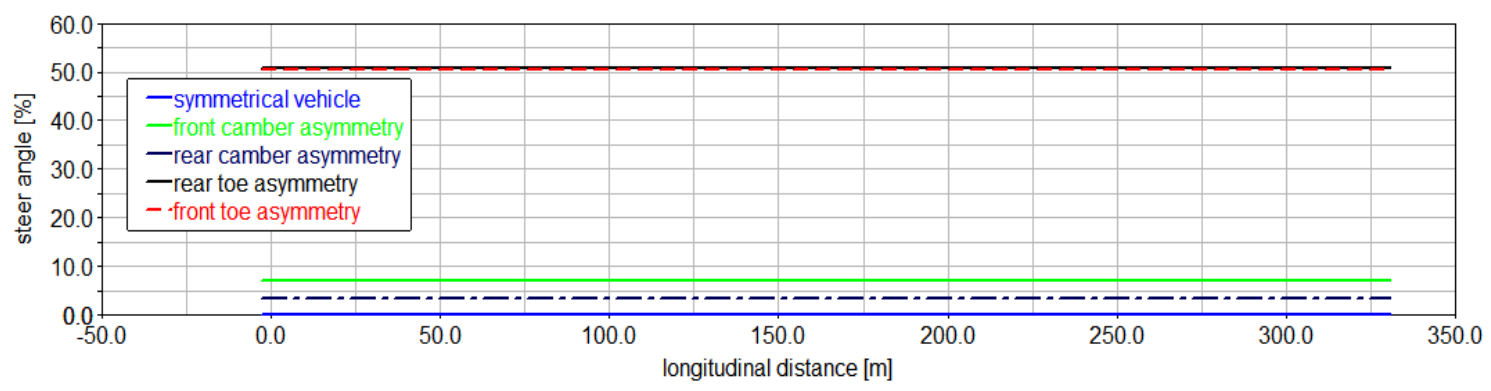

(b)

Figure 7. Effect of asymmetric camber and toe angles on lateral deviation (a) and steering wheel rotation (b).

Different ride steer on the two wheels of the rear axle has been simulated by imposing the largest errors on hardpoint positions due to component tolerances (pre-setting process) while keeping camber and toe angles perfectly regulated (perfect static wheel setup). In this case, anomalies occur only in the presence of vertical oscillations of the suspensions, therefore only DL is evaluated as shown in Figure 8(a) and 8(b).

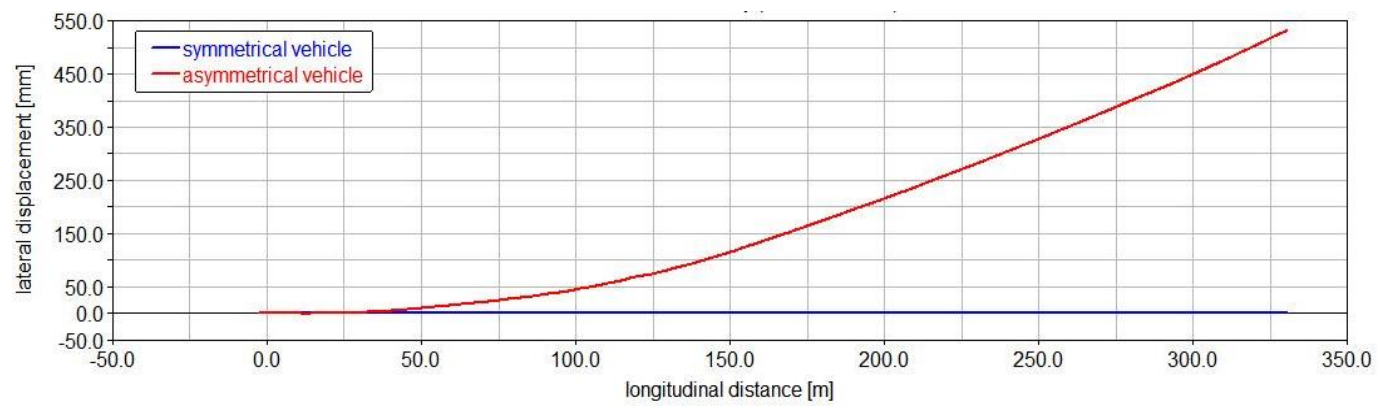

(a)

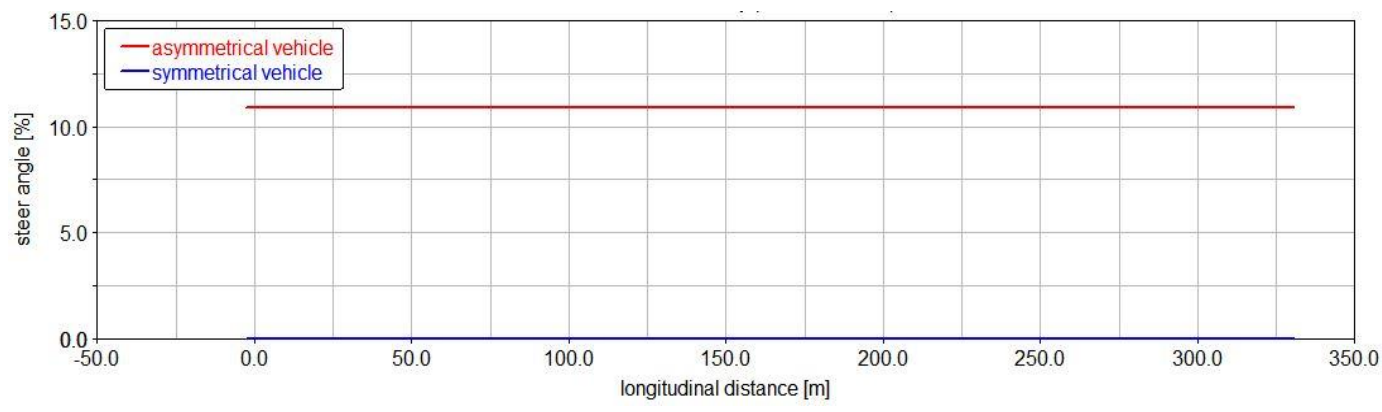

(b)

Figure 8. Effect of asymmetric rear axle ride steers on lateral deviation (a) and steering wheel rotation $(b)$.

During DL, ride steer produces crab angle oscillations, and consequently oscillations of the sideslip angle of the vehicle [18]. The yaw mode of the vehicle (at a natural frequency of about $3 \mathrm{~Hz}$ ) is thus excited, as shown in Figure 9 (Fourier transform of lateral acceleration and sideslip angle), affecting handling and causing discomfort problems. 


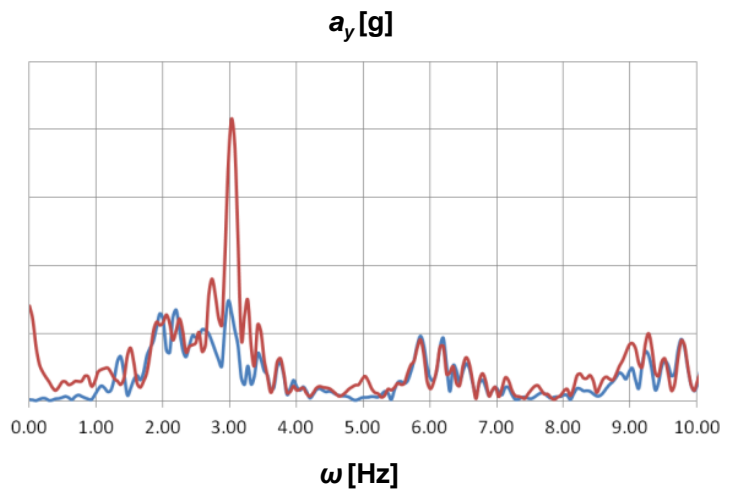

(a)

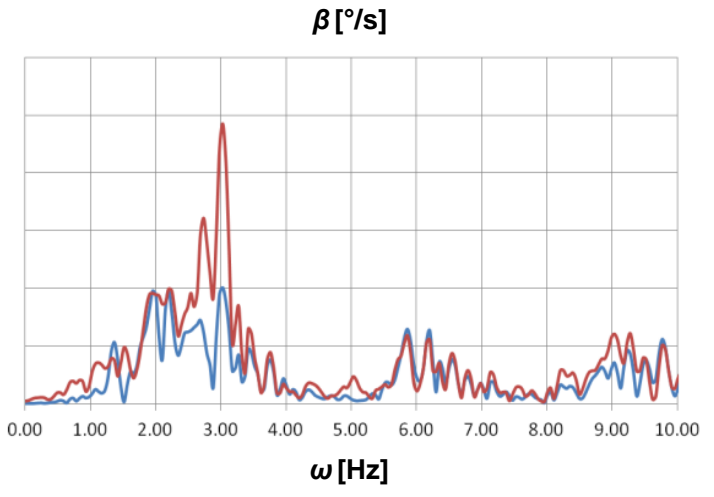

(b)

Figure 9. Fourier transform of lateral acceleration (a) and sideslip angle (b).

\section{METHOD 3: BENCH EVALUATION}

The vehicle assembly process has been improved by considering separately pre-setting bench regulation for reducing rear ride steer, and wheel aligner bench for reducing camber and toe static anomalies.

\section{Pre-setting Bench Evaluation}

Scope of a pre-setting bench is imposing the correct position between rear subframe and uprights; if the components are all in tolerance, once that their relative positions are correct, also the arms will be correctly positioned.

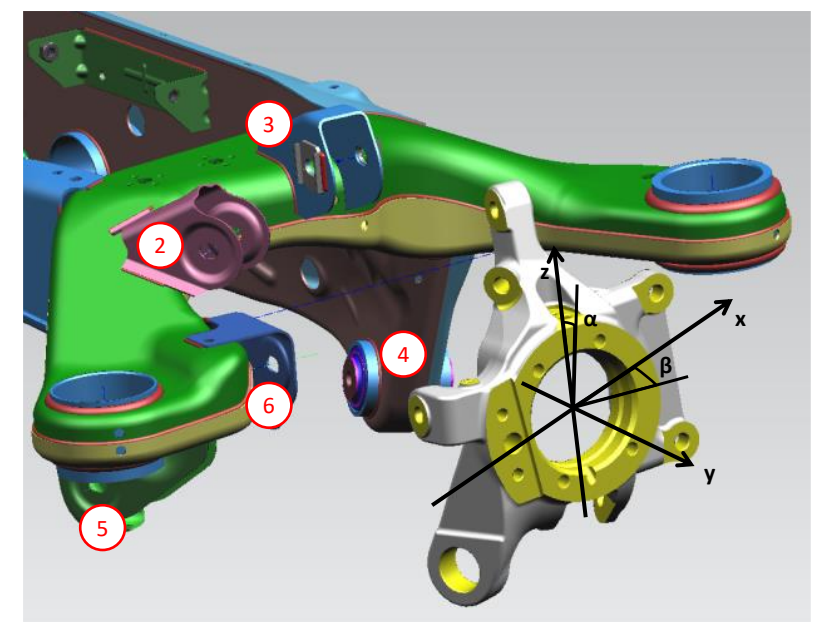

Figure 10. Rear suspension: hardpoints with buttonholes $(2,3,5,6)$. 


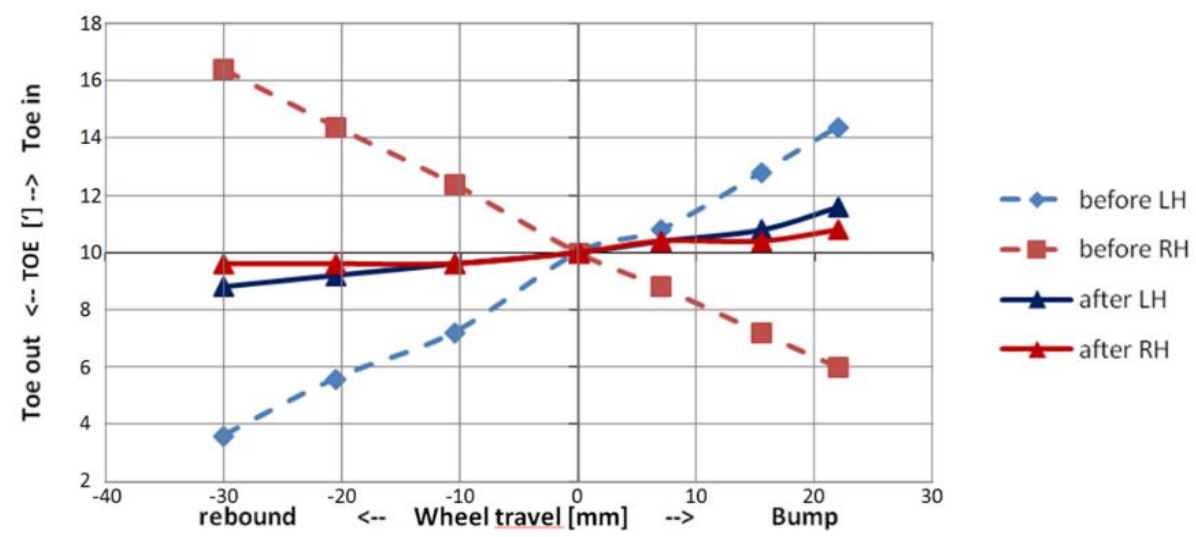

Figure 11. Toe angles ['] comparison before/after pre-setting regulation.

On the pre-setting bench only the position of part of the mechanism members is fixed; with reference to Figure 10 (rear five arms suspension):

i. $\quad$ subframe position (fixed);

ii. $\mathrm{X}$ and $\mathrm{Z}$ upright position (fixed);

iii. Y upright position (variable);

iv. toe and camber angles (variable).

The hardpoints 2, 3, 5, 6 are equipped with buttonholes; among them, the positions of 2 and 5 are fixed, those of 3 and 6 are set up during the final mounting phase, on the wheel aligner bench.

By testing some vehicles affected by DL, strong asymmetries in toe angles were detected, as shown in Figure 11 (dashed lines). To evaluate whether this anomaly was related to the mounting phase or to the component tolerances, the components were disassembled to make again the mounting phase on the pre-setting bench. As a result, ride steer differences were strongly reduced. On some vehicles, the pre-setting analysis was sufficient for reducing DL within acceptable bounds. On other vehicles, however, the presetting analysis was not sufficient to correct the anomaly.

For understanding the effects of both geometry (e.g. definition of kinematic points) and elastic components (e.g. bushings), a virtual model was then updated by measuring all components. Good correlation between virtual simulations and experimental data, as displayed in Figure 12, shows that for the vehicles under analysis the anomaly was caused by structural components. Consequently, a tolerance analysis has been performed for identifying the components out of tolerance.

\section{Wheel Aligner Bench Evaluation}

The regulation of camber and toe angles at the wheel aligner bench is the most delicate phase during a vehicle set up. During this study, two critical aspects have been identified: correct positioning of laser scan columns on the bench, and correct locking of wheels on their supports.

Regarding the first issue, it is important that the laser can scan a great surface of the tyre sidewall, in order to identify the closer point and to define correctly the centreline of the tyre, as shown in Figure 13. By improving sensor positioning, the reading errors of the lasers have been significantly reduced. 


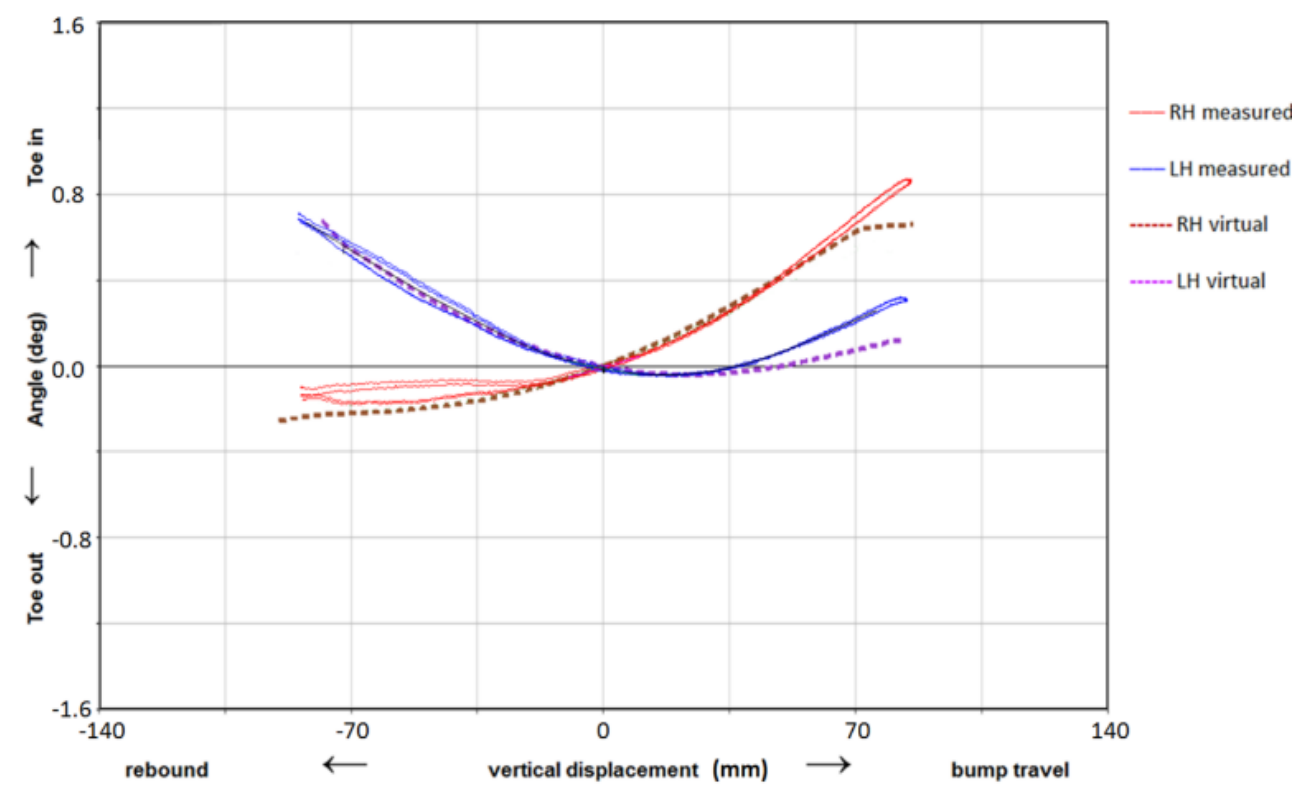

Figure 12. Variation of rear toe $\left[{ }^{\circ}\right]$ angle.

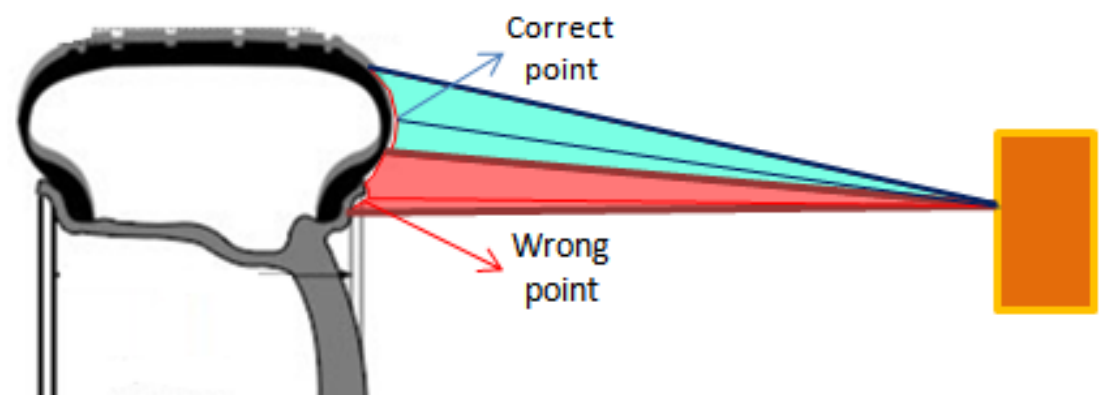

Figure 13. Laser scanning at the wheel aligner bench.

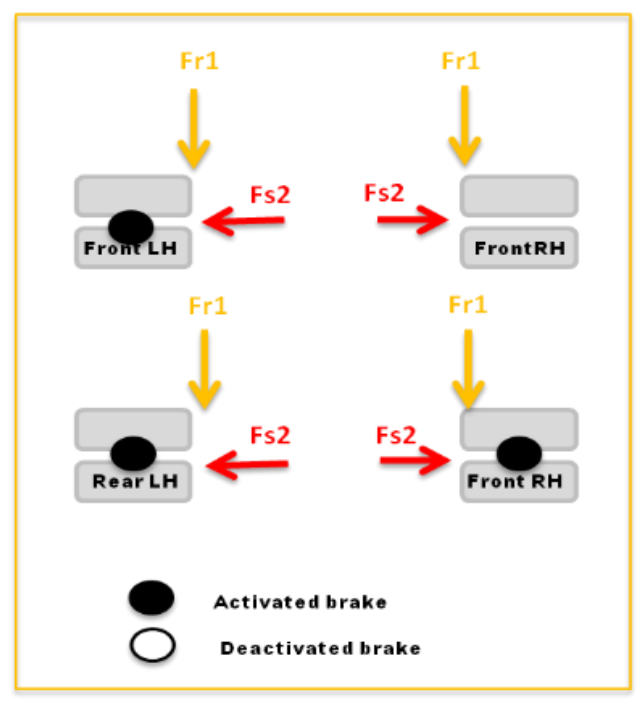

(a)

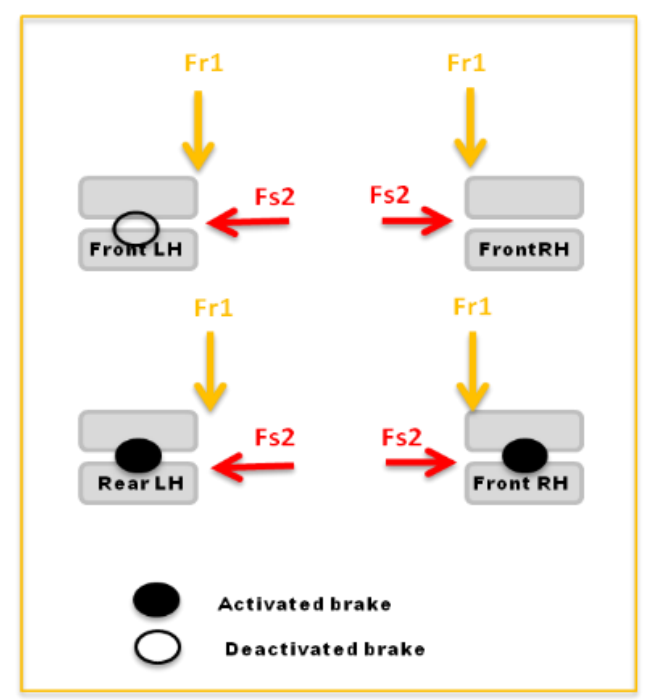

(b)

Figure 14. Front suspensions set up phase at the wheel aligner bench. 
The last phase which has been analysed is locking of wheel supports. Ideally the vehicle should be completely free, for reducing to zero bushing preloads; in practice the vehicle is locked for improving safety and reducing set up time. The improved procedure consists of locking the rear wheels while regulating the front wheels (as shown in Figure 14(b), and then locking one side of the vehicle for regulating the opposite rear wheel. This corrects the existing procedure, in which the front left plate was always locked as shown in Figure 14(a), with the disadvantage of increasing preload on front bushings.

\section{RESULTS AND DISCUSSION}

The virtual analysis put in evidence that PTS and SWM are mainly related to the set-up phase of wheel angles. Both anomalies can be explained by the contribution that vehicle geometry gives to the sideslip angle. Front camber angles influence PTS, while front and rear toe angles influence SWM. The main cause of DL has been identified with (rear) ride steer, which in turn can be generated by components out of tolerances or/and pre-setting variation.

By improving all the set up process (pre-setting bench and wheel aligner bench), on a whole production line the number of vehicles affected by PTS, SWM and DL was strongly reduced: from $3 \%$ down to about $1 \%$ of the production, as reported in Table 1 (where the percentages reported in columns A and B are referred to two parallel sub-lines in which the main production line is split for pre-setting and wheel aligner benches). Clearly, further improvements are still needed for correcting the anomalies of the residual $1 \%$ of vehicles.

Table 1. Percentages of vehicles affected by PTS, SWM and DL.

\begin{tabular}{lccc}
\hline & Total production & $\mathrm{A}(\%)$ & $\mathrm{B}(\%)$ \\
\hline Before improvement & 3.05 & 5.17 & 0.92 \\
After improvement & 1.09 & 1.25 & 0.92 \\
\hline
\end{tabular}

The plot in Figure 15 presents a comparison of front and rear crab angles before and after process improvement, where especially the front crab angle variation has been strongly reduced. The yellow area identifies the bounds for acceptable values (in terms of handling behaviour) of front and rear crab angles. In the diagram, the points on the first and third quadrant diagonal (equal front and rear crab angles) are acceptable in terms of handling behaviour (the vehicle travels on a straight trajectory); actually, even points close to these diagonals are still acceptable (green area).

\section{CONCLUSION}

In this study three relevant handling anomalies affecting passenger cars travelling on a straight path have been considered, namely 'pull to side', 'steering wheel misalignment' and 'drift leeward', in the specific case of vehicles with front Double wishbone suspension and rear Five arms suspension.

After analysing the vehicle assembly process in terms of errors and tolerances to identify which parameters can actually generate these handling irregularities, sensitivity analysis by multibody virtual modelling, and subsequent correlation with experimental data from presetting and wheel aligner benches, provided sufficient information for setting tolerance thresholds, able to keep the handling anomalies under study within acceptable bounds. 
Virtual simulation analysis put in evidence that pull to side and steering-wheel misalignment are mainly related to the set-up phase of wheel angles. Both anomalies can be explained by the contribution that vehicle geometry gives to the sideslip angle. Front camber angles influence pull to side, while front and rear toe angles influence steering wheel misalignment. Main cause of drift leeward has been identified with (rear) ride steer, which in turn can be generated by components out of tolerances and pre-setting variation. As a result of this analysis, the total number of vehicles affected by 'pull to side', 'steering wheel misalignment' and 'drift leeward' on a whole production line was strongly reduced, from $3 \%$ down to about $1 \%$ of the overall production. Further improvements are still needed for correcting the anomalies of the residual $1 \%$ of vehicles.

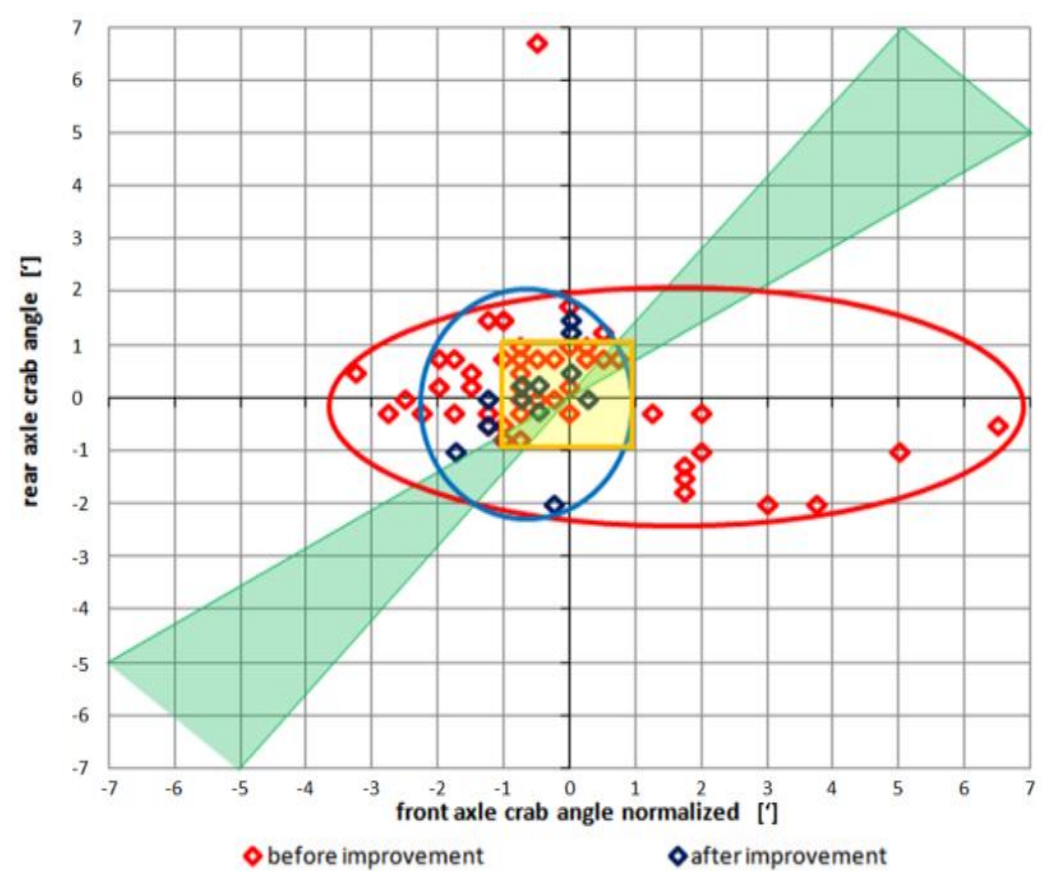

Figure 15. Crab angle ['] variation analysis.

\section{REFERENCES}

[1] Topping RW. Tire Induced Steering Pull. SAE Technical Paper: 750406; 1974.

[2] Matyja FE. Steering pull and residual aligning torque. Tire Science and Technology, 1987, 15 (3): 207-240.

[3] Lindenmuth BE. Tire conicity and ply steer effects on vehicle performance. SAE Technical Paper: 740074; 1974.

[4] Pottinger M. Tire/Vehicle Pull: An introduction emphasizing plysteer effects. Tire Science and Technology, 1990, 18(3): 170-190.

[5] Mundl R, Fischer M, Wajroch M, et al. Simulation and validation of the ply steer residual aligning torque induced by the tyre tread pattern. Vehicle System Dynamics, 2005, 43(1): 434-443.

[6] Yamazaki S, Fujikawa T, Suzuki T, Yamaguchi I. Influence of wheel alignment and tire characteristics on vehicle drift. Tire Science and Technology, 1998, 26(3): 186-205.

[7] Lee JH. Analysis of tyre effect on the simulation of vehicle straight line motion. Vehicle System Dynamics, 2000, 33(6): 373-390. 
[8] Mastinu G, Lattuada A, Matrascia G. Straight-ahead running of road vehicles analytical formulae including full tyre characteristics. Vehicle System Dynamics, 2018, online December 2018.

[9] Kim YS, Jang DY. Optimization of geometric dimension \& tolerance parameters of front suspension system for vehicle pulls improvement. Transactions of the Korean Society of Mechanical Engineers A, 2009, 33(9): 903-912.

[10] Murari TB, Lima DM, Zebende GF et al. Vehicle steering pull: from product development to manufacturing. Product Management \& Development, 2016, 14 (1): 22-31.

[11] Cho YG. Steering pull and drift considering road wheel alignment tolerance during high-speed driving. International Journal of Vehicle Design, 2010, 54(1): 73-91.

[12] Park KS, Heo SJ, Kang DO. Robust design optimization of suspension system considering steering pull reduction. International Journal of Automotive Technology, 2013, 14(6): 927-933.

[13] Aalizadeh B, Asnati A. Integrated bees' algorithm and artificial neural network to propose an efficient controller for active front steering. International Journal of Automotive and Mechanical Engineering, 2016, 13(2): 3476-3491.

[14] Kajiwara S. Improvement in steering performance by push - pull operation in car driving. International Journal of Automotive and Mechanical Engineering, 2018, 15(1): 4919-4934.

[15] Vannucchi de Camargo F, Fragassa C, Pavlovic A, Martignani M. Analysis of the Suspension Design Evolution in Solar Cars. FME Transactions, 2017, 45(3): 394404.

[16] Pacejka HB. Tyre and Vehicle Dynamics. Oxford: Butterworth-Heinemann; 2006.

[17] Matschinsky W. Rad and off - road vehicle system dynamics. Boca Raton (FL): CRC Press Taylor \& Francis Group; 2014. Chapter 21, Suspension Systems: 727768.

[18] Schiehlen W. Road and off-road vehicle system dynamics. Boca Raton (FL): CRC Press Taylor \& Francis Group; 2014. Chapter 2, Vehicle models and equations of motion: $13-44$. 